\title{
EDIFICATE
}

I Congreso de Escuelas de Edificación y Arquitectura Técnica de España València, 4 y 5 de noviembre de 2021

Escuela Técnica Superior de Ingeniería de Edificación

Universitat Politècnica de València

Doi: https://doi.org/10.4995/EDIFICATE2021.2021.13441

\section{Adaptación metodológica de la docencia de la asignatura Matemáticas II del Grado en Arquitectura Técnica de la UPV debida a la Pandemia}

\section{Methodological adaptation of the teaching of Mathematics II subject in Technical Architecture Degree of the UPV due to the Pandemic}

\author{
Juana Cerdán Soriano ${ }^{a}$, Màrius Fullana Alfonso ${ }^{a}$ y David Soler Fernández ${ }^{a}$ \\ ${ }^{a}$ Dep. de Matemàtica Aplicada. Universitat Politècnica de València, Camí de Vera s/n 46022 València \\ jcerdan@mat.upv.es, mfullana@mat.upv.es, dsoler@mat.upv.es
}

\begin{abstract}
This article presents the work carried out to adapt the subject Mathematics II in the Technical Architecture degree of the Polytechnic University of València (UPV) to the confinement conditions due to the COVID-19 pandemic during the second semester of the 2019-20 academic year. Some conclusions and mechanisms used during this period that are considered useful in nonpandemic conditions are also presented.
\end{abstract}

Keywords: mathematics, teaching methodologies, online training, technological resources, evaluation

\section{Resumen}

En este artículo se expone el trabajo realizado para adaptar la asignatura Matemáticas II en el grado de Arquitectura Técnica de la Universitat Politècnica de València (UPV) a las condiciones de confinamiento por la pandemia de la COVID-19 durante el segundo cuatrimestre del curso 2019-20. Se presentan también algunas conclusiones y algunos mecanismos utilizados durante este período que se consideran útiles en condiciones sin pandemia.

Palabras clave: matemáticas, metodologías docentes, formación online, recursos tecnológicos, evaluación. 


\section{Introducción}

La asignatura Matemáticas II consta de 6 créditos repartidos en 3.5 créditos de teoría y 2.5 créditos de prácticas todos ellos impartidos de manera presencial en clases de: 1) teoría seminario, 2) teoría de aula, 3) práctica informática y 4) práctica de aula. A continuación, se detallan las nueve unidades didácticas en que se distribuye la materia impartida:

1. Cálculo diferencial en varias variables

2. Introducción a las ecuaciones diferenciales

3. Estadística descriptiva

4. Introducción a la probabilidad

5. Variables aleatorias discretas

6. Variables aleatorias continuas

7. Distribuciones muestrales

8. Estimación

9. Pruebas de hipótesis

La calificación de la asignatura Matemáticas II se obtiene mediante la media ponderada resultante de los cuatro actos de evaluación siguientes:

1. Primera prueba conjunta, escrita de respuesta abierta, con un peso entorno al $35 \%$, proporcionalmente a la materia impartida. Se entiende por conjunta el hecho de que todos los grupos realizan la misma prueba.

2. Segunda prueba conjunta, con un peso complementario del anterior hasta completar un $70 \%$. Esta prueba, como la primera conjunta, es escrita de respuesta abierta.

3. Pruebas de evaluación continua y/o trabajos académicos, con un peso del $30 \%$. Estas pruebas dependen del profesorado de cada grupo.

4. En caso de que la calificación de la asignatura sea inferior a 5 o tenga opción a evaluación curricular, el alumnado puede optar a realizar un examen de recuperación de una y solo una de las pruebas conjuntas.

La asignatura es impartida por los tres profesores firmantes de este artículo.

En el curso 2019/20 las clases de Matemáticas II del grado de Arquitectura Técnica en la UVP comenzaban el 27 de enero y acababan el 29 de mayo. Hasta el momento del confinamiento el 14 de marzo del 2020, se había impartido la mitad de la materia y el profesorado ya había realizado o propuesto parte de la evaluación continua. A partir del 14 de marzo la docencia pasó a ser virtual y el sistema de evaluación se ajustó a las nuevas circunstancias. Conviene expresar que se realizó un gran esfuerzo por parte de la comunidad educativa para seguir en esta línea, con adaptación inmediata tanto del alumnado como del profesorado, destacando el reto que esto supuso también para los autores, ya que el sistema presencial era diametralmente opuesto al nuevo y no se contaba con niguna experiencia. La coordinación del profesorado se realizó por videoconferencias constantes y exigió un sobreesfuerzo que se hizo con un alto sentido de responsabilidad y voluntad. 
En este trabajo se describe cómo se adecuó toda la docencia de Matemáticas II a esta situación de emergencia. Se realiza un análisis de su desarrollo y resultados. También se presentan las principales conclusiones y aquellas nuevas prácticas que se ha considerado oportuno implementar en el curso actual.

\section{Objetivos}

Tras el confinamiento el 14 de marzo de 2020, la docencia en la UPV pasó totalmente a modo virtual. Para la impartición de la materia ya se disponía de una plataforma virtual que proporciona la UPV Ilamada "PoliformaT". Cada asignatura tiene su propio espacio reservado en esta plataforma exclusivamente para el alumnado matriculado y el profesorado que la imparte. En la docencia presencial (anterior a la pandemia) ya se hacía un uso habitual del citado espacio virtual en esta asignatura, consistente en: 1) La publicación de material didáctico como apuntes, ejercicios resueltos, exámenes de cursos pasados, etc. 2) Anuncios de cualquier incidencia, convocatorias de exámenes, sus soluciones una vez realizados, publicación de notas, etc. 3) Comunicación con el alumnado. 4) Realización de tareas.

En la nueva fase creada por la situación del estado de emergencia y el confinamiento, el uso de las nuevas tecnologías, como ya se ha comentado, ha sido fundamental.

El objetivo de esta comunicación es la descripción del trabajo realizado en nuestra asignatura en la situación de emergencia y la exposición de todo lo aprendido. Se hace un análisis y se extraen unas conclusiones que sirven para hacer propuestas de cara al futuro, algunas de las cuáles ya se han implementado. Aprender de la experiencia y más en situaciones excepcionales es una de las características que nos definen como individuos y como seres sociales y así se constata en lo aquí expuesto.

\section{Desarrollo de la innovación}

Se describe ahora la adaptación de la docencia a esta situación. Se dedica una sección aparte al sistema de evaluación porque se tuvo que hacer un cambio radical que se sumó al gran esfuerzo que se realizó en el aspecto docente por no ser el sistema anterior virtual sino totalmente presencial.

\subsection{Las clases virtuales}

Se decidió utilizar la plataforma "PoliformaT" para explicar cada paso que se daba inmediatamente y de la manera más clara posible. De esta forma, el alumnado tenía claro qué hacer en cada momento y los medios de que disponía para ello. Así por ejemplo, al final de cada semana se publicaba un anuncio con la planificación de la semana siguiente. El Apéndice I muestra como ejemplo de comunicación el anuncio de una convocatoria de 
examen en que se puede apreciar cómo se intentó tener en cuenta cualquier mínimo detalle que pudiera ayudar y clarificar las dudas que pudiesen surgir.

En las carpetas de recursos de PoliformaT asociadas a los grupos de docencia se publicaron, nada más entrar en el estado de emergencia, ficheros pdf correspondientes a los temas que faltaban por impartir de la asignatura (versados sobre estadística). Cada fichero contenía tanto la teoría del tema (totalmente desarrollada y con ejemplos de cada concepto resueltos con todo detalle), como una colección de enunciados de problemas de consolidación junto con sus soluciones. El objetivo de esta publicación temprana era que el alumnado que lo desease pudiera avanzar en el estudio de la materia, según su programación en casa. Adicionalmente a los ficheros de los temas, se publicaron enlaces a vídeos en internet de corta duración donde se explicaban de manera sencilla conceptos puntuales de cada tema. Especial mención merecen los vídeos del repositorio de la UPV conocidos como polimedia, de los que, a modo de ejemplo, se citan dos: uno con la introducción de la variable aleatoria normal (UPV[Media]. Distribución normal) y otro con una explicación sencilla de la estimación, a través de un intervalo de confianza, de la media de una población conocida la desviación típica (UPV[Media]. Estimación).

Asimismo, previo aviso y de modo pautado y ajustado a los horarios de clase habituales (para dirigir el tiempo de aprendizaje de cada apartado), se publicaron en las carpetas de PoliformaT videos con voz en off, en los que se explicaban exhaustivamente todos los conceptos y se resolvían paso a paso los ejemplos correspondientes. Se pretendía que en el horario habitual de clase el alumnado visionase estos vídeos en su casa, pudiendo repetir las veces que considerase oportuno cualquier parte de la grabación para intentar entender todos los conceptos y pasos dados. Se puede considerar que las únicas diferencias con la clase presencial eran el no visionado del profesorado y la imposibilidad de realizar preguntas de forma inmediata al mismo. Pero esta segunda diferencia quedaba subsanada como se explica en el párrafo siguiente.

En los horarios habituales de clase, el profesorado estaba pendiente del correo electrónico corporativo, de manera que cualquier alumno/a o grupo de alumnado que quisiese realizar una consulta al profesorado, sólo tenía que enviarle un correo con el asunto CONSULTA DE invitada CLASE MAT-II, y automáticamente el profesorado invitaba a todo el grupo a conectarse a la plataforma TEAMS, para resolverle la duda de forma inmediata. Esta es una plataforma virtual de videoconferencias del soporte "MS Office" cuya licencia tiene contratada la UPV. Ello permitía al resto del alumnado que estuviera atento a la plataforma, por un lado escuchar e incluso participar de la conversación existente, aunque originalmente la consulta no fuera suya y, por otro, ponerse en "lista de espera", de manera que cuando acabara la consulta anterior, podía realizar la suya propia sin necesidad de escribir un correo previo.

Cuando se consideraba oportuno, según las consultas recibidas y particularmente para explicar resoluciones de problemas tipo, previo aviso con suficiente antelación, el profesorado usaba el horario de clase presencial para impartir una clase online a través de la plataforma TEAMS. Por la naturaleza de la materia, la resolución de problemas se iba exponiendo paso a paso a través de diapositivas en Powerpoint, de manera que se incitaba al alumnado a 
intervenir e indicar el paso siguiente en la resolución, así como a la búsqueda de datos en tablas o a la realización de cálculos sencillos, lo que permitía comprobar que se entendían los conceptos. El Apéndice II muestra una figura con la secuenciación de las diapositivas mostradas para la resolución de un problema tal como se visionaba por el alumnado durante la clase virtual por TEAMS.

Por último, en las carpetas de recursos de PoliformaT se fue publicando material adicional, como enunciados de exámenes anteriores, tablas y otros documentos que se consideraban convenientes según el desarrollo de la docencia y las necesidades particulares del alumnado.

\subsection{Las tutorías}

Puesto que la tutoría presencial era inviable (la UPV estaba cerrada para cualquier actividad no esencial), se optó por dos métodos de asistencia individual al alumnado.

- $\quad$ Si las dudas o preguntas eran concretas y breves se atendían por correo electrónico. Se recomendaba al alumnado que las hicieran llegar al profesorado por dicho medio, en días laborables de lunes a viernes, siendo atendidas lo antes posible en jornada laboral y por orden de recepción del correo. Las preguntas debían exponerse con la mayor claridad posible, y venir acompañadas, si resultaba necesario, de documentación (foto, docx, etc.).

- $\quad$ En caso contrario, se podía optar por tutorías, lo más parecido a las tradicionales, pero obviamente a través de la plataforma de vidoconferencia TEAMS. Una vez recibido el correo electrónico con la petición de tutoría por parte de un alumno, una alumna o un grupo del alumnado, el profesor concertaba día y hora en el grupo de TEAMS correspondiente.

\subsection{Cambio del sistema de evaluación}

Antes de presentar el método de evaluación propuesto cabe destacar que antes de pasar al sistema de enseñanza virtual ya se había impartido la mitad del contenido teórico y práctico de la asignatura. Los temas que quedaban de estadística no contenían conceptos teóricos de excesiva complejidad y sólo requerían de cálculos sencillos (con ayuda de calculadora) y el uso de tablas.

Se propuso el siguiente sistema de evaluación que fue aprobado por la Comisión Académica del Título de Grado y que anuló el que constaba hasta ese momento en la guía docente de la asignatura:

Una evaluación de clase con un peso del 10\%. Como ya se comentó en la Sección 1, a fecha de 14 de marzo ya se había realizado o propuesto en la asignatura parte de la evaluación continua correspondiente al $30 \%$ inicial de la nota, con lo que este peso del $10 \%$ se asignó a esta parte de la evaluación continua.

Una evaluación conjunta (todo el alumnado de la asignatura) con un peso del $90 \%$ repartido en 3 exámenes ( $30 \%$ cada uno) que se realizó a través de la plataforma PoliformaT con la 
herramienta "Exámenes". Cabe destacar que ello implicó una dedicación importante en horas por parte del profesorado de esta asignatura, sólo para el estudio y manejo de esta herramienta en la configuración de un examen, en las diferentes modalidades de preguntas (tipo test, con resultado numérico, subida de ficheros, etc.), y en lo que se refiere a la introducción de las preguntas en baterías, particularmente en esta asignatura, por la necesidad de incluir fórmulas y tablas en las mismas. Para este fin fue muy útil el curso de preparación de exámenes con esta herramienta a través de la página web habilitada por la UPV (Universitat Politècnica de València. Curso de Realización de Exámenes en PoliformaT).

\section{Estructura de los exámenes}

Antes de describir los exámenes realizados, destacar que al tener grupos de docencia en castellano, valenciano e inglés se tuvieron que redactar todas las preguntas en los 3 idiomas.

El primer examen se realizó el 22/04/2020. La materia evaluada se correspondía con la parte de cálculo (temas 1 y 2). Se optó por las preguntas de tipo test pues nos permitía utilizar LaTex [Lamport,L. 1994] para la introducción de las expresiones matemáticas tan necesarias en los temas de cálculo.

El examen constaba de 17 preguntas de tipo test con 4 opciones que se seleccionaban de forma aleatoria de una batería de 10 preguntas cada una de ellas. Además, se configuró de forma que las diferentes opciones de cada pregunta también se aleatorizaban. En la Figuras 1 y 2 se presenta un ejemplo de pregunta tipo test sobre la materia del primer y segundo tema, respectivamente. Además, en la Figura 1 puede verse parte de la configuración del examen. En la parte superior aparecía la información relativa al número de la pregunta y la puntuación de la misma y en la parte inferior aparecen 4 botones. Por una parte los botones de Anterior-Siguiente que le permiten avanzar y retroceder durante la realización del examen y por otra parte los botones de Guardar-Salir.

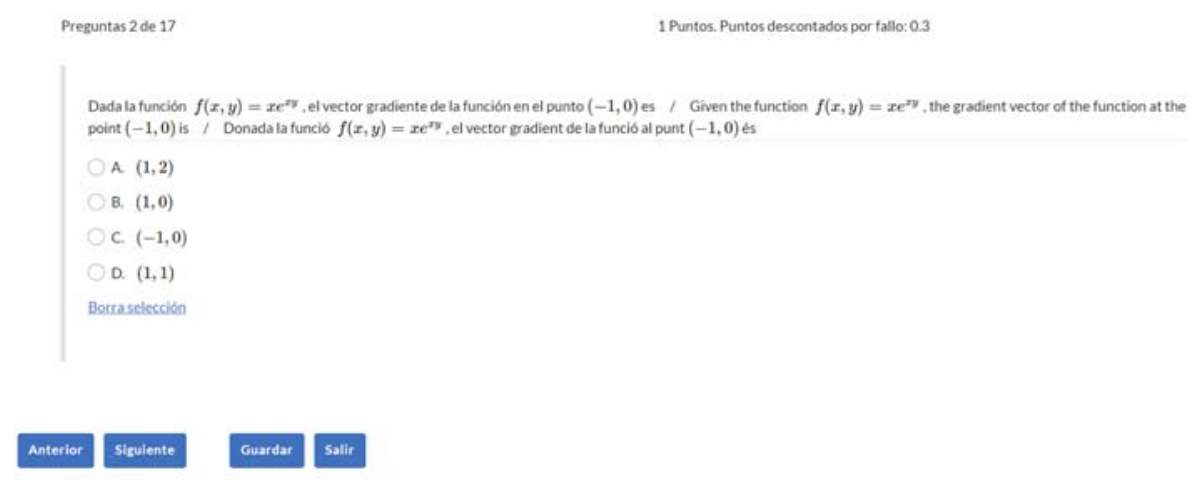

Fig. 1. Pregunta test Tema 1

La herramienta "Examen" no la habíamos utilizado hasta entonces. Se envió información a los alumnos sobre la ruta a seguir para acceder a los exámenes y se publicó una semana 
antes del examen oficial una prueba con preguntas tipo test para que los alumnos pudiesen practicar y así eliminar una parte del estrés del examen.

La duración del examen fue de 2 horas y se prepararon dos versiones del examen cambiando el orden. Tras realizar el examen el alumno recibía la nota y podía comparar su solución con la correcta como puede verse en la Figura 3.

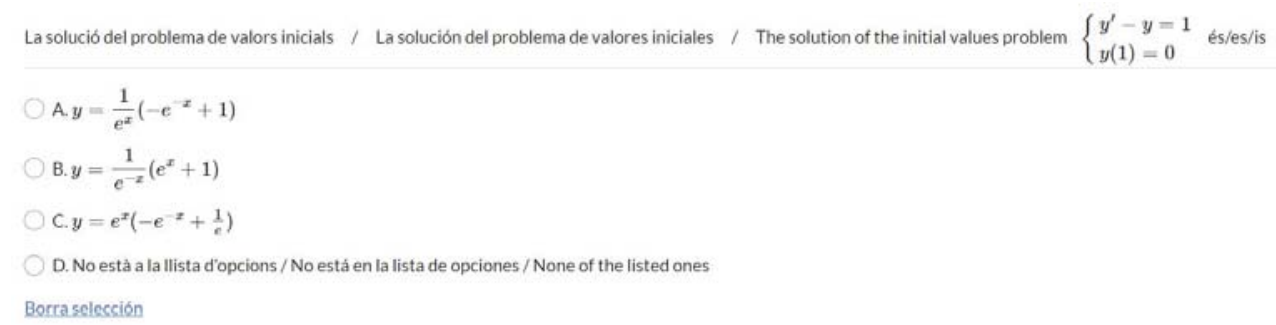

Fig. 2. Pregunta test Tema 2

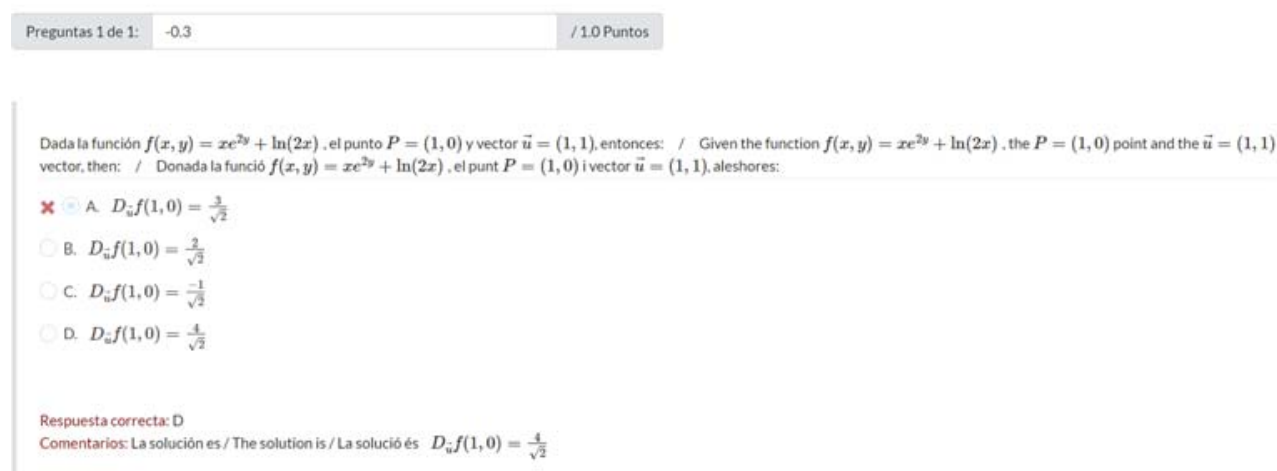

Fig. 3. Ejemplo de revisión de pregunta via PoliformaT.

El segundo examen se realizó el 20/05/2020. La materia evaluada se correspondía con los tres primeros temas de estadística. Este examen se configuró combinando preguntas de tipo numérico (hay que escribir la respuesta en un recuadro) y de tipo test (hay que marcar una opción de las cuatro posibles).

El examen se estructuró en 4 bloques:

$1^{\text {er }}$ bloque: Pregunta de tipo numérico con 4 apartados seleccionada de forma aleatoria de una batería de 20 preguntas

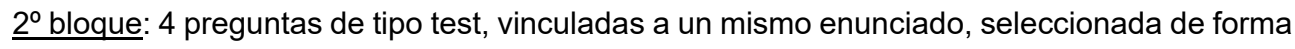
aleatoria de una batería de 20 preguntas 
$\underline{3^{e r} \text { bloque: }}$ Pregunta de tipo numérico seleccionada de forma aleatoria de una batería de 20 preguntas

$44^{\circ}$ bloque: Dos preguntas, una de tipo numérico y otra de tipo test seleccionadas de forma aleatoria baterías de 20 preguntas

La pregunta final de cada bloque consistía en subir el documento pdf (máximo 20MB cada fichero) resultado de escanear la foto del folio o folios donde aparecían desarrolladas las preguntas de dicho bloque. El objetivo, como se indicó al alumnado, era disponer del documento para una posible revisión para subsanar algún error como introducir mal la solución en el recuadro, error al marcar la opción en las preguntas tipo test, nunca por errores de cálculo. La falta del documento anulaba la pregunta.

Una diferencia importante respecto a la configuración del primer parcial es que en este examen no se permitió volver atrás en ninguna pregunta. Siempre hacia adelante. Se les informó de un procedimiento sencillo para convertir una foto en un documento pdf y, para que pudiesen practicar la subida de documentos y que valorasen el tiempo que tardaban, se publicó un simulacro de examen breve. La Figura 4 presenta un ejemplo de pregunta numérica del Tema 3. En cada recuadro el alumnado debía introducir la solución. Para evitar errores en la corrección debidos a que se podían realizar cálculos con diferente precisiones, en la configuración de la pregunta se establecía un intervalo de solución. En la Figura 5 se presenta una pregunta tipo numérico sobre la materia del Tema 5. La Figura 6 muestra la pregunta en la que se pedía que se subiese el fichero manuscrito de trabajo.

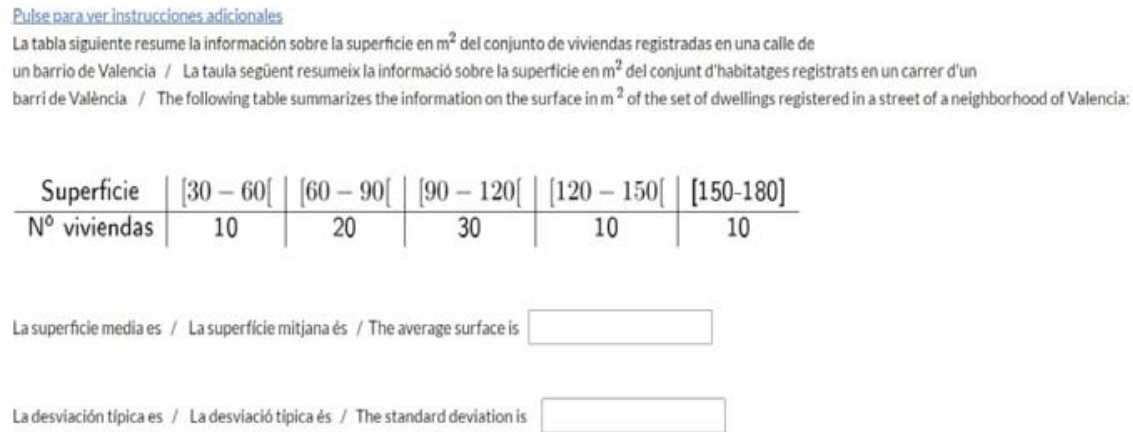

Si una inmobiliaria está interesada sólo en el 20 por ciento de las viviendas de mayor superficie de la calle, ¿cuál es la superficie minima de dichas viviendas? / Si una immobiliària está interessada només en el 20 per cent dels habitatges de major superficie del carrer, quina ès la superficie minima d'aquests habitatges? / If a real estate agency is interested in only 20 percent of the houses with the largest surface area on the street, what is the minimum surface of said houses?

Fig. 4. Pregunta test Tema 3 


\author{
Pulse para ver instrucciones adicionales \\ En una fàbrica de rajoles ixen 4 trencades de mitjana en 1 dia. Si la distribució que segueix és de Poisson, calcula la probabilitat que isquen trencades: \\ En una fábrica de ladrillos salen 4 rotos de media en 1 día. Si la distribución que sigue es de Poisson, calcula la probabilidad de que salgan rotos: \\ In a tile factory there are 4 breaks on average in 1 day. If the following distribution is from Poisson, calculate the probability that they will break: \\ 1) 7 en 2 dies / 7 en 2 días / 7 in 2 days: \\ 2) Com a màxim 10 en 2 dies / como máximo 10 en 2 días / maximum 10 in 2 days: \\ 3) Almenys 9 en 2 dies / al menos 9 en 2 días / at least 9 in 2 days: \\ 4) Més de 3 i menys de 6 en $1 \mathrm{dia} /$ más de 3 y menos de 6 en 1 día / more than 3 and less than 6 in 1 day:
}

Fig. 5. Pregunta test Tema 5

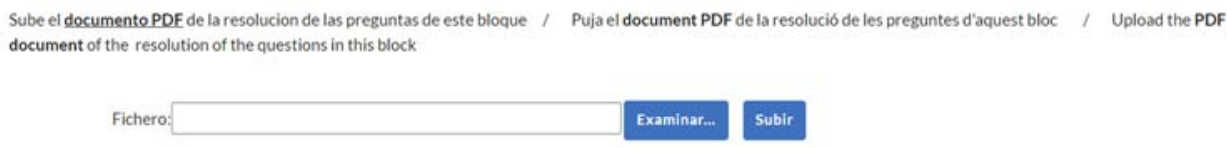

Fig. 6. Pregunta subir el fichero de trabajo

La duración del examen fue de 2 horas y se prepararon dos versiones del examen cambiando el orden de las preguntas. En este caso el proceso de corrección fue un poco más largo pues se tenía que comprobar que los ficheros con las resoluciones se habían subido y comprobar que se correspondía con las soluciones introducidas.

El tercer examen se realizó el día 08/06/2020. La materia evaluada se correspondía con los últimos temas de estadística. Este examen se configuró de forma similar al anterior, combinando preguntas de tipo numérico y de tipo test. Antes de cambiar de bloque debían subir el documento pdf que acreditaba la autoría de las soluciones introducidas.

El examen se estructuró en 3 bloques:

$\underline{1^{\text {er }} \text { bloque: }}$ Pregunta de tipo numérico con 4 apartados seleccionada de forma aleatoria de una batería de 18 preguntas

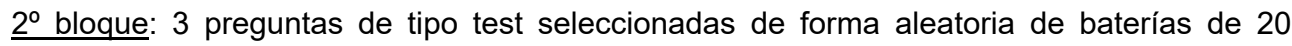
preguntas cada una

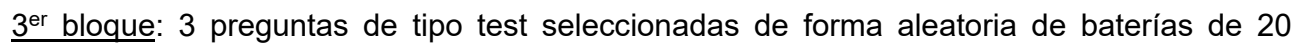
preguntas cada una

La Figura 7 muestra un ejemplo de pregunta numérica del tema 6 y la Figura 8 muestra una pregunta tipo test del tema 8. 


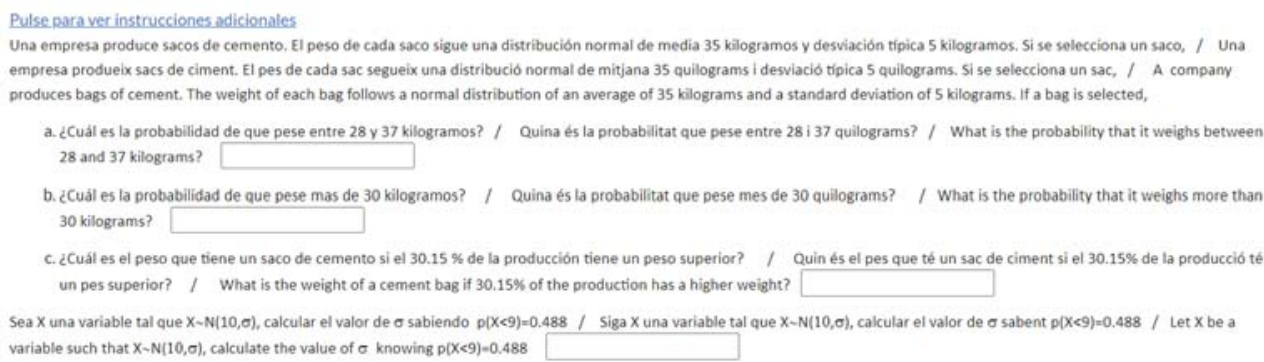

Fig. 7. Pregunta numérica Tema 6

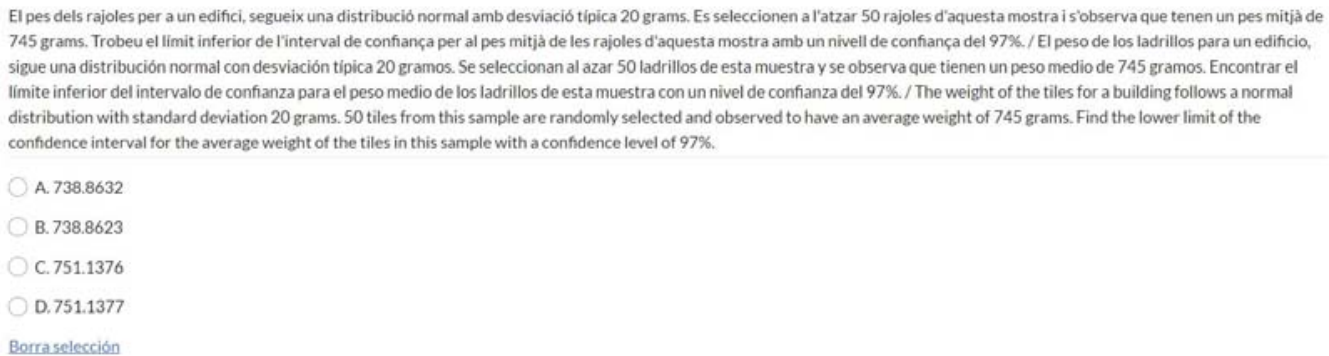

Fig. 8. Pregunta test Tema 8

La duración del examen fue de 1:30h y se prepararon dos versiones del examen cambiando el orden de las preguntas.

Como se indicó en el sistema de evaluación se programó un examen de recuperación. Podía optar a esta recuperación el alumnado cuya calificación de la asignatura fuese inferior a 5 y sólo se podía recuperar uno de los parciales. El examen se realizó el día 19/06/2020.

Se prepararon 3 exámenes de recuperación, uno por cada parcial, a los que se presentaron 2,6 y 21 alumnos, respectivamente. Se varió ligeramente la configuración y estructura de los exámenes. En los parciales 1 y 2 se reutilizaron las preguntas de las baterías y en el parcial 3 se crearon nuevas baterías. En concreto:

Recuperación $1^{\text {er }}$ parcial: Constaba de 12 preguntas tipo test y subir la resolución.

Recuperación $2^{\circ}$ parcial: Constaba de 4 bloques, cada uno de ellos con preguntas numéricas con 4 apartados. Debían subir la resolución.

Recuperación $3^{\text {er }}$ parcial: Constaba de 2 bloques. El primer bloque era un problema de 6 apartados y el segundo bloque estaba formado por 4 preguntas numéricas. Debían subir la resolución. 


\section{Resultados}

Se presentan resultados en dos aspectos diferentes. Por un lado en lo que concierne al número de aprobados y su comparativa con años anteriores y por otro, respecto de la aplicabilidad y aprovechamiento de la experiencia obtenida a una situación de no pandemia, con presencialidad total.

\subsection{Cambio del sistema de evaluación}

Los resultados obtenidos en cada parcial se presentan en la Tabla 1. En las primeras columnas se presentan los resultados de aprobados frente a presentados de cada parcial y la última columna recoge los resultados finales de la asignatura. Aquí se observa una mejora considerable de los resultados del segundo y tercer parcial respecto del primero. Esto puede ser debido a tres causas. La primera es que históricamente en la parte de cálculo se obtienen siempre peores resultados que en la parte de estadística. La segunda es que en la realización del primer examen los alumnos estaban confinados y no en los otros. La tercera es que por ser la primera vez que se realizaban este tipo de examen, quizás no supieron distribuir el tiempo de forma adecuada además de la dificultad de enfrentarse a un tipo de examen totalmente nuevo.

Tabla 1. Resultados de aprobados

\begin{tabular}{|l|c|c|c|c|}
\cline { 2 - 5 } \multicolumn{1}{c|}{} & 1 $^{\text {er }}$ parcial & $\mathbf{2}^{\mathbf{0}}$ parcial & 3 $^{\text {er }}$ parcial & Matemáticas II \\
\hline Presentados & 98 & 91 & 83 & 103 \\
\hline Aprobados & 26 & 53 & 45 & 60 \\
\hline$\%$ Aprobados & 26.53 & 58.24 & 54.21 & 58.25 \\
\hline
\end{tabular}

En la Figura 9 se muestra una comparativa del rendimiento del alumnado presentado el curso 2019-20 con los tres cursos anteriores. Como se observa en dicha figura, los medios informáticos disponibles y la responsabilidad y la voluntad de superación de toda la comunidad educativa de la asignatura de Matemáticas II ha hecho que los resultados docentes se hayan mantenido como en el nivel superior de los cursos anteriores, sin la emergencia social vivida. Este hecho pone en valor el inmenso trabajo realizado. 


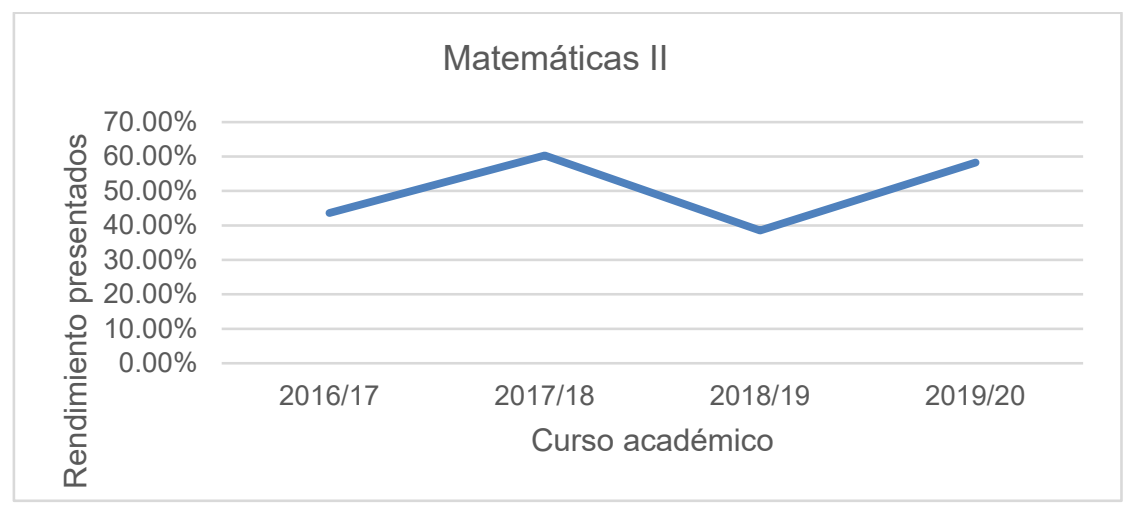

Fig. 9. Rendimiento del alumnado presentado

\subsection{Aplicabilidad de la experiencia en situación de normalidad}

Afortunadamente, la asignatura Matemáticas II se imparte en el segundo cuatrimestre, por lo que las condiciones de la pandemia en el curso 2020-21 en la Comunitat Valenciana o País Valencià han permitido que tanto el sistema de enseñanza como de evaluación en la escuela donde se imparte el grado de Arquitectura Técnica sea mayoritariamente presencial. Se ha vuelto por tanto al sistema de evaluar conjuntamente en prueba escrita y presencial el $70 \%$ de la asignatura y el otro $30 \%$ en evaluación continua grupal.

No obstante, la experiencia excepcional del curso pasado, y en particular la ingente cantidad de problemas acumulados en la herramienta "Exámenes" de la plataforma PoliformaT (ya detallada en la Sección 3) y que dieron lugar a millones de combinaciones de exámenes diferentes, ha permitido al profesorado de esta asignatura replantearse algunos aspectos en la impartición de la misma el curso 2020-21, y previsiblemente en cursos venideros. Notar que la herramienta Exámenes permite que los problemas puedan ser reagrupados o repartidos en diferentes baterías, de forma que se pueden generar exámenes de diferentes características o de diferente cantidad de materia a los realizados en curso 2019-20.

A continuación, se detallan algunas variaciones o aprovechamientos de la experiencia del curso anterior, respecto del sistema usado en cursos anteriores:

- $\quad$ Dado que la distancia social (1.5 metros) y el uso de mascarilla se ha seguido manteniendo durante todo el curso 2020-21, se ha combinado el sistema de tutoría tradicional presencial con el sistema usado el curso pasado a través de la plataforma TEAMS, así como con el uso de correo electrónico para dudas muy concretas. El objetivo de la tutoría solicitada, así como la cantidad de alumnado previsiblemente presente en dicha tutoría son la base para determinar si una tutoría debe ser presencial o vía TEAMS. En cualquier caso, por los comentarios recibidos, cabe destacar que el alumnado valora mucho el poder hablar con el profesorado viéndose las caras sin necesidad de desplazarse de su casa a propósito para una tutoría. Téngase en cuenta que las tutorías no deben solaparse con el horario de clases, y eso hace que, por ejemplo, alumnado del grupo de tarde deba en ciertas ocasiones acudir 
a la UPV a tutorías en horario de mañana. Así pues, el profesorado de esta asignatura considera que las tutorías a través de TEAMS son beneficiosas para los dos actores de éstas, previo acuerdo, por lo que su idea es seguir usándolas en cursos próximos.

- $\quad$ Algunos de los actos de evaluación no conjunta, que implican el 30\% de la nota, han sido realizados este curso 2020-2021 a través de la herramienta "Exámenes" de PoliformaT, aprovechando las baterías de problemas almacenadas el curso pasado. Por citar un ejemplo concreto, en la realización de un acto de evaluación de prácticas con ordenador con el programa Mathematica (Wolfram Mathematica), si tradicionalmente se ponía el mismo examen a todo el grupo, ahora cada estudiante es consciente de que tiene un examen diferente del resto, el cual realiza a través de PoliformaT, aunque de manera presencial en el aula. Ello facilita enormemente la labor de control de un examen en un aula de ordenadores y en el que todo tipo de apuntes está permitido, así como el poder realizar en casos justificados el examen desde fuera del aula, con control a través de TEAMS. Además, se añade la ventaja para el profesorado de que la ingente tarea de generación de problemas de examen el curso anterior hace que en este curso, y probablemente en algunos cursos venideros, no necesite generar nuevos exámenes.

- $\quad$ Conectando con el final del ítem anterior, incluso algunos de los problemas de los enunciados de los exámenes conjuntos han sido extraídos (y modificados si se consideró oportuno) de las baterías generadas el curso pasado. De alguna forma, se puede concluir que el profesorado "recupera" parte del tiempo extra invertido durante la situación excepcional del curso 2019-2020.

\section{Conclusiones}

De la adaptación metodológica de la docencia de la asignatura de Matemáticas II del Grado en Arquitectura Técnica de la UPV debida a la Pandemia se pueden extraer las siguientes conclusiones, detalladas en la Sección 4:

- Esta adaptación no supuso una variación sustancial en el porcentaje de aprobados del alumnado respecto de cursos anteriores. De hecho igualó práctimente al mejor porcentaje de los tres cursos anteriores.

- La experiencia adquirida por el profesorado y la ingente cantidad de material elaborado le ha hecho introducir cambios dentro del método de enseñanza presencial, que considera mejoras y comodidad tanto para el profesorado como para el alumnado, como son las tutorías vía TEAMS y la realización de exámenes individualizados vía PoliformaT, tanto dentro como fuera del aula, esto último espacialmente para alumnos con dispensa de asistencia y poca disponibilidad horaria. 


\section{Referencias}

Lamport, L. LaTeX: A Document Preparation System : User's Guide and Reference Manual. Addison Wesley, Reading, Mass., EUA, 2a edición, 1994

Universitat Politècnica de València. UPV[Media]. Distribución Normal. https://media.upv.es/\#/portal/video/8b2421d7-6fe4-834d-a555-a9037dc8e66c (Consulta: 13/06/2021).

Universitat Politècnica de València. UPV[Media]. Estimación de la media. https://media.upv.es/\#/portal/video/9a100389-37e3-174a-b229-4d1e7ea57c4b (Consulta: 13/06/2021).

Universitat Politècnica de València. Curso de Realización de Exámenes a través de

PoliformaT. http://personales.upv.es/sanvipui/eufol/eufol.html (último acceso 13/06/2021).

Wolfram, Mathematica. http://www.wolfram.com/mathematica. (Consulta: 02/06/2021).

\section{Apéndice I}

\section{Convocatoria del segundo parcial:}

Apreciados alumnos,

La evaluación del $2^{\circ}$ parcial de Matemáticas II se realizará mediante la herramienta Exámenes de PoliformaT. El examen se abrirá a las 18:30 h del 20 de mayo y constará de 4 bloques. La duración máxima del examen será de 105 minutos, por lo que el examen deberá ser enviado para su corrección antes de las 20:15 h. Todo examen enviado con posterioridad a esta hora será considerado por el sistema como "no presentado".

Hay preguntas de tipo numérico (hay que escribir la respuesta en un recuadro) y de tipo test (hay que marcar una opción de las cuatro posibles). Recordar que en las de tipo test cada pregunta incorrecta descuenta 1/3 de su valor, por lo que se aconseja contestar sólo si se tiene un alto grado de seguridad de acertar, nunca al azar.

En la última pregunta de cada bloque deberéis subir el documento PDF (máximo 20MB cada fichero) resultado de escanear la foto del folio o folios en los que hayáis resuelto las preguntas planteadas en dicha parte con el nombre Problema_1, Problema_2, etc. Es importante que se respete la denominación de los ficheros para que sepamos qué contiene cada uno de 
ellos. Si no se sube el fichero de una parte la puntuación en dicha parte será de 0 y no se admitirán envíos de ficheros por otros medios.

Como se especificó en el primer parcial, necesariamente deberéis realizar el documento manuscrito sobre folio/s blanco, a una cara y con tinta azul o negra, nunca con lápiz (no se lee bien). No importan los tachones. Puesto que disponéis de todo el material incluyendo fórmulas y tablas, la revisión en base a los documentos PDF subidos solo se realizará en casos excepcionales debidamente justificados y no por errores de cálculo.

Importante: durante la realización del examen, una vez contestada una pregunta (o en su defecto, pasando a la siguiente pregunta sin contestar la actual) no se podrá volver a contestar, ni revisar, ni cambiar preguntas anteriores. La configuración del examen/test no permitirá volver atrás en ninguna pregunta. Siempre hacia adelante. Así pues, deberéis gestionar el tiempo que dedicáis a cada bloque para poder acceder con suficiente tiempo a todas ellas. Por razones del sistema, no deberéis estar más de 44 minutos en una misma parte sin guardar la pregunta y pasar a la siguiente, ya que el sistema dará por caducada la sesión, no responsabilizándose el sistema de posibles fallos en la reconexión. Recordar guardar a medida que vayáis contestando las preguntas.

Respecto del uso de decimales, cuando se pida una probabilidad, ésta siempre se calculará usando las tablas que se os ha proporcionado, y por tanto manteniendo siempre los 4 decimales, no aceptándose resultados con truncamientos o redondeos a menos de 4 decimales. Por otro lado, si en otras operaciones no relacionadas con las tablas de variables aleatorias, la calculadora proporciona más de 4 decimales, se deberán mantener como mínimo 4 decimales a lo largo de todas las operaciones, de manera que, como mucho, el error cometido en el resultado sea de unas diezmilésimas. Dicho de otro modo, si estáis seguros de que habéis realizado las operaciones correctamente y vuestro resultado difiere sólo en el cuarto decimal respecto de una de las respuestas y se aleja mucho de las restantes, lo lógico es que esa sea la respuesta correcta.

Para que podáis practicar la subida de documentos vamos a abrir un simulacro de examen breve (nada que ver con el oficial) que constará de 2 bloques y que estará activo durante el lunes día 18 de mayo de $9 \mathrm{~h}$ a $13 \mathrm{~h}$.

Saludos

Juana Cerdán

Profesora responsable de Matemáticas II 


\section{Apéndice II}

Figura con la secuenciación de las diapositivas mostradas para la resolución de un problema a través de la plataforma TEAMS:

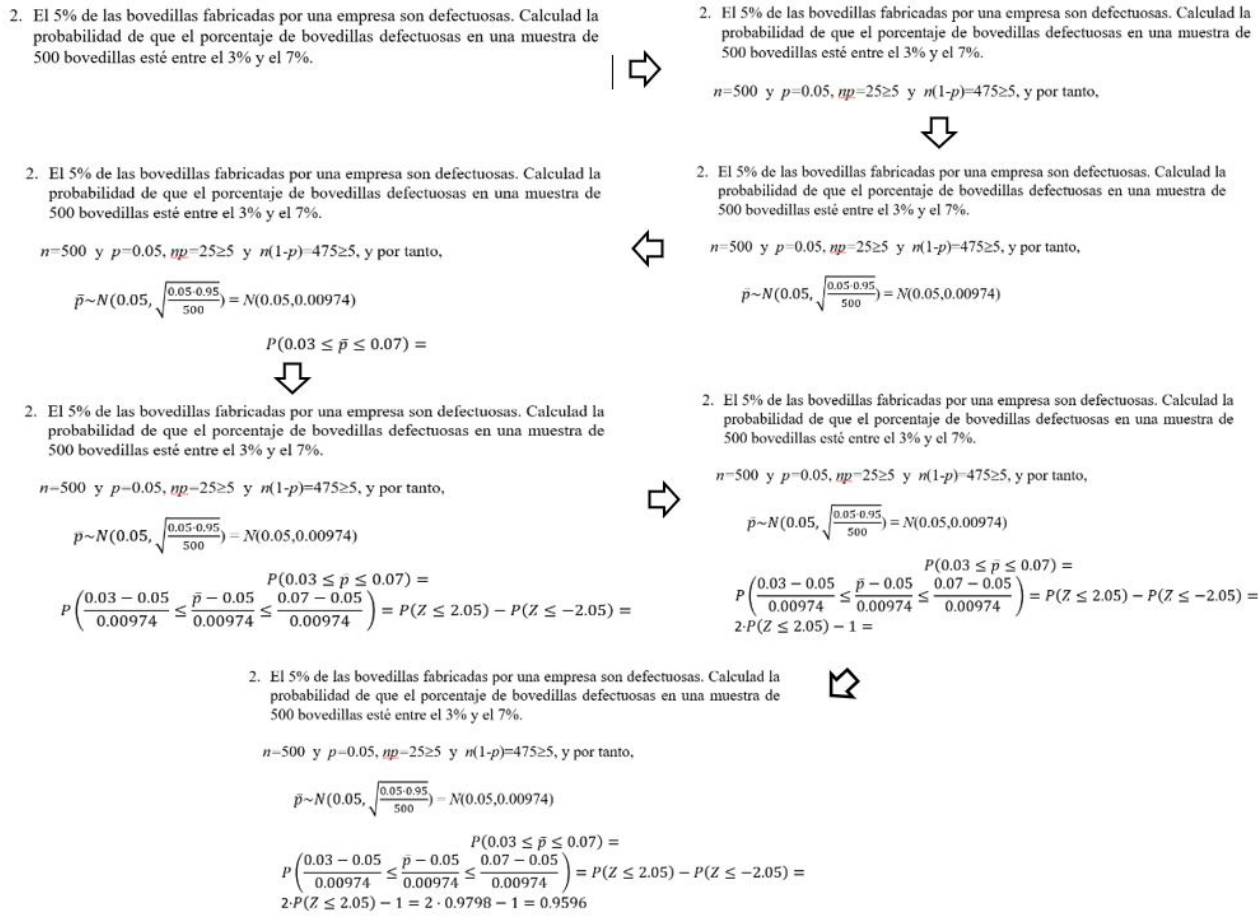

